Aufgrund des aus dem Bundesstaatsprinzip folgenden Gebots zur wechselseitigen Rücksichtnahme ist die einseitige Kündigung jedoch die ultima ratio ${ }^{74}$.

Angesichts der aktuellen Tendenzen zu bundesweit einheitlichen Wahlperioden von fünf Jahren und der Rechtslage scheint die Zusammenlegung der Wahltermine also durchaus realisierbar. Bund und Länder an einen Tisch zu bekommen und eine gemeinsame Linie zu erarbeiten, dürfte sich in der Praxis aber nicht einfach gestalten.

74 Zuvor müssen insbesondere Gespräche und Verhandlungen über eine Vertragsanpassung gescheitert sein. Das ist kaum vorstellbar. Zur Bedeutung des Prinzips der Bundestreue für Staatsverträge siehe Jost Pietzcker, Landesbericht Bundesrepublik Deutschland, in: Christian Starck (Hrsg.), Zusammenarbeit im Bundesstaat, Baden-Baden 1988, S. 17, S. 65 ff.; Hans Schneider, Verträge zwischen Gliedstaaten im Bundesstaat, in: VVDStRL, Heft 19 (1961), S. 1, S. 14 ff.

\title{
Gewichtetes Wahlrecht: Hilfsmittel für Neugliederungen und angemessene Repräsentation im Bundesstaat?*
}

\author{
Julia Gieseler
}

Wahlen sind eine Grundvoraussetzung moderner liberal-pluralistischer Demokratien. Als wesentliches Element politischer Partizipation gilt das allgemeine, direkte und geheime sowie überwiegend auch das gleiche Wahlrecht. In der Regel garantiert es die Verfassung. Dabei hat der Grundsatz gleicher Wahl die größte Bedeutung für die Ausgestaltung von Wahlsystemen $^{1}$ : Im Mehrheitswahlrecht muss jeder Stimme der gleiche Zählwert zukommen, im Verhältniswahlrecht zur Umsetzung des Gebots der Wahlrechtsgleichheit auch der gleiche Erfolgswert. Vor allem die Wahlkreisgröße und -einteilung ist für das gleiche Wahlrecht wichtig. So können politische Parteien (Gerrymandering) oder Landesteile und Bevölkerungsgruppen (Grundmandatsklausel, nationale Minderheiten ${ }^{2}$ ) bevorzugt werden, wenn nicht immer die gleiche Zahl von Wahlberechtigten auf ein Mandat entfällt.

Für die hilfreiche Unterstützung und Anregungen bei der Erstellung des Beitrags danke ich Erich Röper.

1 „Das Gebot der Wahlgleichheit ist kein Kriterium für die Auswahl eines Wahlsystems, sondern bildet die maßgebliche Grundlage für seine Ausgestaltung": Wolfgang Schreiber, Handbuch des Wahlrechts zum Deutschen Bundestag. Kommentar zum Bundeswahlgesetz, 6. Auflage, Köln 1998, S. 99. Zur Wahlrechtsgleichheit für viele Norbert Achterberg / Martin Schulte, in: Horst Dreier (Hrsg.), Grundgesetz, Bd. 2, München 2005, Rdnrn. 121 ff. zu Art. 38.

2 Die Zulässigkeit von Überhangmandaten (zuletzt BVerfGE 95, S. 335 ff.), Grundmandatsklausel (dazu BVerfGE 95, S. 408 ff.) und Privilegierung der Parteien nationaler Minderheiten wird hier ausgeklammert. Zu den Parteien nationaler Minderheiten Erich Röper, Up ewig ungedelt? - Zum Wahlrecht der dänischen Minderheit in Schleswig-Holstein, in: NordÖR 2003, S. 391 ff., Everhard Holtmann, Dürfen die das, wo sie doch Dänen sind? Über den Umgang mit Macht und Minderheiten in Deutschland, in: ZParl, 36. Jg. (2005), H. 3, S. 616 - 629, und die BVerfGBeschlüsse vom 17. November 2004, in: NordÖR 2005, S. 19 ff., und 14. Februar 2005, 2 BvL $1 / 05$. 
In Deutschland darf die Wahlberechtigtenzahl eines Wahlkreises nach Artikel 38 GG, dem Bundeswahlgesetz, der ständigen Rechtsprechung des Bundesverfassungsgerichts sowie Artikel 28 Abs. 1 GG in den Ländern, Kreisen und Kommunen grundsätzlich nicht um mehr als 15 Prozent vom Durchschnitt aller Wahlkreise abweichen. Nur ausnahmsweise ist beispielsweise zur Einhaltung von Landesgrenzen oder wegen ständiger Migrationsprozesse eine Abweichung bis zu einer Obergrenze von 25 Prozent tolerierbar ${ }^{3}$; wird sie überschritten, ist eine Neueinteilung der Wahlkreise vorzunehmen.

In anderen europäischen Staaten werden andere Vorstellungen entwickelt: Durch eine spezifische Wahlkreiseinteilung in dünn besiedelten Gebieten wird der Grundsatz gleichen Wahlrechts aus politischen Gründen oder zur Garantie politischer Repräsentation bewusst verletzt oder die „gewollte“ Verletzung der Wahlrechtsgleichheit gestattet, damit diese Bevölkerungsteile größeres Stimmgewicht haben - ein Modell, das Anklang hätte finden können bei dem Fusionsversuch von Berlin und Brandenburg, der 1996 in Brandenburg mehrheitlich abgelehnt wurde. Zu diskutieren ist, ob eine stärkere Repräsentation dünn besiedelter Gebiete in Deutschland mehr Zustimmung etwa bei betroffenen Bevölkerungen für Länderfusionen bewirken könnte.

\section{Wahlkreiseinteilung und Wahlrechtsgleichheit in Europa: ausgewählte Beispiele}

Um die Wahlrechtsgleichheit zu garantieren, muss „vermieden werden, daß die Wählerschaft eines Wahlkreises im Verhältnis zu der eines anderen Wahlkreises bei der Repräsentation durch Wahlkreisabgeordnete bevorzugt oder benachteiligt wird“4. Dies wird nicht in allen europäischen Staaten und auch nicht bei den Wahlen zum Europäischen Parlament (EP) eingehalten.

In den meisten europäischen Demokratien wird das Wahlgebiet in Mehrpersonenwahlkreise unterschiedlicher Größe eingeteilt. Die auf einen Wahlkreis entfallenden Mandate (beziehungsweise im Falle Frankreichs auf ein Département) richten sich nach der jeweiligen Bevölkerungszahl (beispielsweise Belgien und Griechenland), der Zahl der dort lebenden Staatsangehörigen (beispielsweise Finnland, Frankreich und Österreich), der Zahl der Wahlberechtigten (beispielsweise Vereinigtes Königreich, Schweden und Spanien) oder nach einer Kombination mehrerer Faktoren wie Einwohnerzahl und Bevölkerungsdichte (beispielsweise Dänemark und Norwegen). Zur Wahrung der Wahlrechtsgleichheit existieren Bestimmungen zur regelmäßigen Anpassung der Wahlkreise beziehungsweise der Anzahl der Mandate, die auf einen Wahlkreis entfallen, wobei Abweichungen teilweise dadurch gerechtfertigt werden, dass kleinere oder abgelegene Gebietseinheiten bevorzugt

3 Das gilt nicht für Mandatskontingente: Zu den verfassungsrechtlichen Anforderungen an die Erfolgswertgleichheit bei der Mandatsverteilung auf die Wahlbereiche Bremen und Bremerhaven Urteil des BremStGH vom 5. November 2004, in: NordÖR 2005, S. 485 ff.

4 Wolfgang Schreiber, a.a.O. (Fn. 1), S. 159 f. Gleichzeitig muss die Wahlkreiseinteilung regelmäßig überprüft und an durch Migrationsprozesse veränderte Verhältnisse angepasst werden, „entweder (im Falle von Einerwahlkreisen) durch die geografische Neufestlegung der Wahlkreisgrenzen oder (im Falle von Mehrpersonenwahlkreisen) durch Modifikation der Zahl der Mandate, die auf die Wahlkreise entfallen“. Dieter Nohlen, Wahlrecht und Parteiensystem, 5. Auflage, Opladen 2007, S. 86 . 
werden. ${ }^{5}$ Im Folgenden soll dies (neben dem EP) an vier Beispielen, der Wahlkreiseinteilung im Vereinigten Königreich, Spanien, Frankreich und Norwegen verdeutlicht werden ${ }^{6}$, in denen vom deutschen Wahlrecht klar abgewichen wird. Vor dem Hintergrund zum Teil nur schwer zugänglicher Materialien in der jeweiligen Landessprache können hier nur diese Beispiele ausführlich behandelt werden, die sich jedoch unter anderem hinsichtlich der geografischen Lage und der Wahlsysteme unterscheiden. Dabei wurde versucht, auf die vier unterschiedlichen Kategorien einzugehen, wobei Norwegen ein Sonderfall ist, in dem sowohl die Bevölkerungszahl als auch die flächenmäßige Größe eines Verwaltungsbezirks bei der Verteilung der Mandate relevant ist.

\subsection{Vereinigtes Königreich}

Im britischen relativen Mehrheitswahlsystem ist die politische Repräsentation für England, Schottland, Wales und Nordirland gewichtet. 1944 wurde für jeden der Landesteile eine permanente Wahlkreiskommission eingesetzt. Diese Boundary Commissions sollten regelmäßig die Wahlkreisgrößen überprüfen und dem Parlament Änderungsvorschläge machen, das diese annehmen oder ablehnen, aber nicht ergänzen oder ändern kann. Bei den Vorschlägen zur Wahlkreiseinteilung hatten die Boundary Commissions zwei Vorgaben zu berücksichtigen: Die Wahlberechtigtenzahl pro Wahlkreis sollte um nicht mehr als 25 Prozent vom nationalen Durchschnitt abweichen, und gemäß dem Besitzstand von 1944 sowie der Stärke der regionalen Repräsentation gab es feste Mandatszahlen beziehungsweise eine Mindestzahl von Mandaten für die vier Landesteile. Schottland sollte mit mindestens 71, Wales mit mindestens 35 Abgeordneten im Unterhaus vertreten sein. Nordirland wurden nur zwölf Mandate zugestanden, da es mit dem Stormont ein eigenes Parlament hat. ${ }^{8}$

Die Vorgaben ließen sich nicht verbinden. Daher wurde 1958 festgelegt, die 25-ProzentQuote nur noch auf die Wahlkreiseinteilung innerhalb der vier Landesteile anzuwenden. Damit wurde ein Verstoß gegen die Wahlrechtsgleichheit zugunsten der Gebietsrepräsentation in Kauf genommen. ${ }^{9}$ So betrug die durchschnittliche Wahlberechtigtenzahl 1983 in England 66.000, in Wales 59.000, in Schottland 54.000 und in Nordirland 61.000. ${ }^{10} 2004$ klafften die Zahlen in England und Schottland mit $70.000 \mathrm{zu} 53.000$ gar noch weiter aus-

5 Vgl. Max-Planck-Institut für ausländisches öffentliches Recht und Völkerrecht, Wahlkreiseinteilung in westlichen europäischen Demokratien, den USA und Kanada, Gutachten für das Bundesverfassungsgericht, in: Zeitschrift für ausländisches öffentliches Recht und Völkerrecht, Bd. 57 (1997), S. $633-674$.

6 Für eine Übersicht der Praxis der weiteren westeuropäischen Länder (mit Ausnahme Portugals) sei verwiesen auf ebenda.

7 Die zeitlichen Vorgaben für die Boundary Commissions wurden mehrfach angepasst. Nach den Regeln von 1944 sollten sie alle drei bis sieben Jahre tätig werden, seit 1958 wurden ihre Berichte alle zehn bis fünfzehn Jahre erwartet; vgl. Dieter Nohlen, a.a.O. (Fn. 4), S. 287. Der Boundary Commissions Act 1986 begrenzte die Zeitspanne auf acht bis zwölf Jahre. Nach der nächsten Wahlkreisüberprüfung, die 2007 beendet sein wird, wird die Aufgabe auf die vom Political Parties, Elections and Referendum Act 2000 gegründete Electoral Commisson übergehen. Vgl. http://www.parliament.uk/works/elections.cfm\#genelec, abgerufen am 5. November 2005.

8 Vgl. Dieter Nohlen, a.a.O. (Fn. 4), S. 287.

9 Vgl. ebenda.

10 Vgl. Pippa Norris, The Politics of Electoral Reform in Britain, in: International Political Science Review, 16. Jg. (1995), S. 65 ff., S. 72. 
einander. ${ }^{11}$ Die Stimme eines schottischen Wählers zählte also mehr als die eines englischen. Schottland, Wales und Nordirland waren in Westminster überrepräsentiert.

Die Mandatsfestlegung wurde durch den 1986 verabschiedeten und 1992 geänderten Parliamentary Constituencies Act nur geringfügig modifiziert. ${ }^{12}$ Auch sperrte sich die seit 1997 amtierende Labour-Regierung gegen eine neue Wahlkreiseinteilung, da sie von den Regelungen aufgrund ihrer Stärke in Schottland und im südlichen Wales sowie wegen der demografischen Entwicklung profitierte, die einen Vorteil von circa 40 Mandaten für die Labour-Partei ergaben. ${ }^{13}$ Erst die Umsetzung der im Scotland Act von 1998 vorgesehenen Bestimmungen vor der Unterhauswahl von 2005 veränderte die Größe der Wahlkreise merklich und verringerte die Zahl schottischer Parlamentssitze von 72 auf 59. Nachdem sich die Mehrheit der Bürger Schottlands im September 1997 für die Einführung eines Regionalparlaments mit eigenen Kompetenzen ausgesprochen hatte, regelte der Scotland Act in Part V unter anderem, dass die Größe der schottischen Unterhauswahlkreise jener der englischen Wahlkreise anzugleichen sei; ihre Durchschnittsgröße beträgt in England und Schottland nun 69.935 Wahlberechtigte. Dass die Vorgaben des Scotland Act nicht zu den Unterhauswahlen 2001, sondern erst 2005 umgesetzt wurden, lässt vermuten, dass die Labour-Regierung so lange wie möglich von der „alten“ Wahlkreiseinteilung profitieren wollte, da Schottland schon lange als Labour-Hochburg gilt. Schottland musste die Überrepräsentation in Westminster also nicht aufgrund fehlender Wahlrechtsgleichheit, sondern wegen der Etablierung seines Regionalparlaments aufgeben.

Deutlich wird, dass die Wahlkreiseinteilung aus politischen und geografischen Gründen erfolgt. Den drei Landesteilen Schottland, Wales und Nordirland wird in Westminster eine höhere Repräsentation als England zugesprochen, um sie ans „Mutterland“ zu binden, sie für den Verzicht auf ihre Souveränität zu entschädigen und mehr Einfluss auf die nationalen Entscheidungsträger zu ermöglichen. Wird ihnen allerdings eine größere Autonomie und ein Parlament mit eigenen Kompetenzen zugestanden, schwindet ihr Einfluss im Unterhaus wieder, wie sich am Beispiel Schottlands und dem Scotland Act von 1998 zeigt. $^{14}$ Zudem wird als rechtens anerkannt, einzelnen Regionen eine bessere Vertretung im Parlament zu geben. Daher dürfen aus Gründen der Tradition und Identität einzelne Wahlkreise von der Durchschnittsquote (69.935 Wahlberechtigte) abweichen, etwa der englische Wahlkreis Hexam in der ländlichen Gegend Northumberland (59.600 Wahlberechtigte) oder der schottische Wahlkreis Na h-Eileanan an Iar (etwa 25.000 Wahlberechtigte), der die Western Isles an der Westküste umfasst. ${ }^{15}$

11 Vgl. BBC News, Scottish constituency cull begins, published 2004/12/14, unter http://news.bbc. co.uk/go/pr/fr/-/hi/scotland/4093765, abgerufen am 5. November 2005.

12 Der Parliamentary Constituencies Act verlangt für Wales und Schottland mindestens 35 beziehungsweise 71 Wahlkreise, Nordirland soll nicht mehr als 18 und nicht weniger als 16 Sitze erhalten. In der Praxis erhielten Schottland 72, Wales 38 und Nordirland 17 Sitze.

13 Vgl. Dieter Nohlen, a.a.O. (Fn. 4), S. 288.

14 Die damit verbundene Reduzierung der schottischen Sitze im House of Commons löst zwar nicht die im Vereinigten Königreich regelmäßig diskutierte „West Lothian-Frage“. Sie schwächt die darin beschriebene Anomalie, dass schottische Abgeordnete in Westminster über englische Belange abstimmen können, aber weder sie noch die englischen Abgeordneten dort dies bei ähnlichen Belangen in Bezug auf Schottland können, aber ab. Vgl. James Mitchell, Großbritannien nach der Devolution, in: APuZ, B 47 (2005), S. 26 ff.

15 So Glenn Reed vom Commission Secretariat des britischen Parlaments in einer E-Mail an die Autorin. 


\subsection{Spanien}

Auch in Spanien wird der Gleichheitsgrundsatz bei den Wahlen zum Abgeordnetenhaus (Congreso de los Diputados) verletzt. Die Wähler der kleineren Wahlkreise sind überrepräsentiert. Das Land ist in 52 Mehrpersonenwahlkreise verschiedener Größe eingeteilt, wobei die Wahlkreisgrenzen mit denen der 50 Provinzen und zwei spanischen Exklaven übereinstimmen. Der Congreso hat 350 Abgeordnete, die nach dem Verhältniswahlrecht gewählt werden. ${ }^{16}$ Außer den Exklaven Ceuta und Melilla (74.931 beziehungsweise 68.463 Einwohner 2003), in denen nur je ein Abgeordneter gewählt wird, hat jede Provinz eine Mindestrepräsentation von derzeit drei Abgeordneten. ${ }^{17}$ Die Zahl der weiteren Mandate richtet sich nach der Bevölkerungsgröße der Wahlkreise. Ohne diese Mindestrepräsentation hätte eine Vielzahl dünn besiedelter Provinzen nur einen Abgeordneten, beispielsweise Zamora, Guadalajara, Palencia und Soria, deren Bevölkerungszahl zum Teil weit unter 200.000 liegt.

Durchschnittlich werden 6,7 Abgeordnete pro Wahlkreis gewählt, wobei Madrid und Barcelona mit 35 und 31 Abgeordneten eine krasse Ausnahme bilden; nur hier und in den anderen großen Wahlkreisen Sevilla und Valencia (12 beziehungsweise 16 Abgeordnete) kann das Verhältniswahlrecht seine volle Wirkung entfalten und eine proportionale Verteilung der Mandate gewährleisten. In den kleinen und mittelgroßen Wahlkreisen ist dies kaum möglich. Kleine Parteien haben daher und wegen der in der Verfassung vorgesehenen Sperrklausel von drei Prozent so gut wie keine Chance auf ein Mandat. Nur regional sehr starke Parteien wie die der Basken oder Katalanen können auch in kleineren Wahlkreisen einen Sitz gewinnen. Die Wahlkreiseinteilung bevorzugt vor allem die beiden großen Parteien; unter bestimmten Umständen kann eine Partei „mit annähernd 42\% der Stimmen die absolute Mehrheit der Sitze erringen "18. So erreichten die Sozialisten (PSOE) bei den Wahlen von 1982 mit gut 48 Prozent der abgegebenen Stimmen 57,7 Prozent der Sitze ${ }^{19}$.

Die Wahlkreiseinteilung begünstigt dünn besiedelte, strukturschwache Provinzen. Aragón, Kastilien-La Mancha, Kastilien-León und Extremadura, die vier am dünnsten besiedelten der 17 Regionen (15,5 Prozent der Gesamtbevölkerung), erstrecken sich über 52 Prozent des nationalen Territoriums. Extremadura und Kastilien-La Mancha weisen zudem mit das geringste Einkommen aus unselbständiger Arbeit auf; mit Asturien gab es dort 2003 die niedrigste Erwerbsquote. Die höchsten Erwerbsquoten hatten die dicht besiedelten Regionen Madrid (wo auch das zweithöchste Einkommen erzielt wird) und Valencia, ebenso die Balearen, Kantabrien und die Kanarischen Inseln.

16 Die Verfassung schreibt eine Abgeordnetenzahl von mindestens 300 und höchstens 400 vor; bisher betrug sie bei allen Wahlen 350; vgl. Dieter Nohlen / Andreas Hildenbrand, Spanien. Wirtschaft - Gesellschaft - Politik, 2. Auflage, Wiesbaden 2005, S. 264; Angaben zum spanischen Wahlsystem finden sich ebenda, Angaben zu Wahlergebnissen und Wahlkreisgröße unter http:// electionresources.org/es/index_es.html, abgerufen am 23. Januar 2006.

17 Vgl. Dieter Nohlen, a.a.O. (Fn. 4), S. 100. Während die Abgeordnetenzahl für Ceuta und Melilla mit je einem Abgeordneten festgelegt ist, gibt die spanische Verfassung für die Provinzen nur vor, dass ihnen eine Mindestrepräsentation garantiert sein muss (Art. 68). Über die Höhe der Mindestrepräsentation entscheidet der Gesetzgeber. Sie lag bisher immer bei zwei oder drei Mandaten.

18 Ebenda, S. 358.

19 Vgl. ebenda, S. 359. 
Die Wahlkreiseinteilung und Mandatsverteilung auf die Wahlkreise verhindert die proportionale Repräsentation der Parteien und verletzt das Prinzip der Gleichheit der Wahl, da die Bevölkerung der kleineren Wahlkreise überrepräsentiert ist. So kamen bei den Wahlen 2004 zum Congreso de los Diputados 31.000 Einwohner auf einen Abgeordneten der bevölkerungsärmsten Provinz Soria, hingegen vertrat ein Abgeordneter aus Madrid 171.000 Einwohner. Die Stimme eines Wählers aus Soria hat also ein mehr als fünfmal höheres Gewicht als die eines Wählers aus Madrid. Da aber dieses Wahlsystem von wissenschaftlicher Seite ${ }^{20}$ und vor dem Hintergrund von Spaniens besonderem politisch-historischen Kontext verteidigt wird, ist eine Änderung unwahrscheinlich. ${ }^{21}$ Vermutlich ist sie politisch nicht einmal erwünscht, da das Wahlsystem die zwei großen Parteien begünstigt, ihnen zu einer stabilen Machtbasis verhilft und gleichzeitig den Gegensatz der agrarisch geprägten Binnenräume zu den dicht besiedelten Provinzen durch die stärkere Repräsentation der Bevölkerung der dünn besiedelten Gebiete und ihrer Interessen abzuschwächen versucht.

\subsection{Frankreich}

Auch in Frankreich wird bei den Wahlen zum Abgeordnetenhaus die Wahlrechtsgleichheit durch Bestimmungen verletzt, die eine stärkere Repräsentation dünn besiedelter Gebiete garantieren sollen. Das Land ist bei den Wahlen zur Assemblée Nationale in 577 Einpersonenwahlkreise untergliedert. Der Zahl der Staatsbürger entsprechend werden 570 Mandate auf die 100 Départements verteilt, wobei jedes mindestens durch zwei Mandate repräsentiert wird. Die sieben weiteren Mandate entfallen auf die Überseegebiete. Innerhalb der Départements werden der zugeteilten Mandatszahl entsprechend die Wahlkreise eingeteilt, wobei jeder in etwa gleich viele Bürger umfassen soll. ${ }^{22}$

Die unter Charles de Gaulle 1958 eingeführte Regel der Mindestrepräsentation begünstigt dünn besiedelte, ländliche Regionen. Heute kommen in den bevölkerungsreichsten 25 Départements knapp 115.000 Einwohner auf einen Abgeordneten, in den 25 am dünnsten besiedelten sind es nur 80.000. Im zweiten Wahlkreis des Départements Lozère, dem mit der geringsten Bevölkerung, vertritt ein Abgeordneter 34.374 Einwohner, der bevölkerungsreichste Wahlkreis, der zweite im Département Val-d'Oise, umfasst hingegen 188.200 Einwohner. ${ }^{23}$ Die Stimme eines Wählers des zweiten Wahlkreises der Lozère hat also mehr als fünfmal soviel Gewicht wie die eines Wählers des zweiten Wahlkreises des Val-d'Oise. Diese Ungleichheiten liegen jedoch nicht nur in der Mindestrepräsentation, sondern auch darin begründet, dass die Wahlkreiseinteilung seit 1986 nicht mehr den demografischen Veränderungen angepasst wurde. Die bestehenden Wahlkreisgrenzen und die Verteilung der Mandate auf die Départements gehen auf die Volkszählung von 1982 zurück, obwohl seit den Volkszählungen 1990 und 1999 Zahlen vorliegen, die die Bevölkerungsunter-

20 Vgl. José R. Montero, La reforma del régimen electoral, Madrid 1994; Juan Montabes (Hrsg.), El sistema electoral a debate: veinte años de rendimientos del sistema electoral español (1977-1997), Madrid 1998.

21 Vgl. Dieter Nohlen, a.a.O. (Fn. 4), S. 265.

22 Vgl. Frédéric Rouvillois, Droit constitutionnel, La Ve République, Paris 2001, S. 82.

23 Vgl. Michel Balinski, Le suffrage n'est plus universel, in: Libération, 27. Juli 2005, unter http:// www.math.uni-augsburg.de/stochastik/bazi/Balinski2005b.html, abgerufen am 30. Mai 2007. Die Einwohnerangaben entsprechen der letzten Volkszählung von 1999. 
schiede und Ungleichheit zwischen den Départements deutlich machen und obwohl das Gesetz n 86-825 vom 11. Juli 1986 vorschreibt, dass spätestens nach jeder zweiten Volkszählung eine Neueinteilung der Wahlkreise vorzunehmen sei. ${ }^{24}$ Diese hätte also spätestens zur Wahl der Nationalversammlung im Jahr 2002 erfolgen sollen. Da in Frankreich nicht eine unabhängige Kommission, sondern die Regierung dafür zuständig ist, hängt eine Neueinteilung immer von ihrem politischen Willen ab. ${ }^{25}$ Dieser scheint aber seit Jahren weder bei Konservativen noch bei Sozialisten vorhanden zu sein, trotz einer Forderung des Conseil Constitutionnel im Mai 2003. ${ }^{26}$ Auch bei der Wahl zur Assemblée Nationale im Juni 2007 wurde die Wahlkreiseinteilung von 1986 beibehalten. ${ }^{27}$ Die Ungleichheit der Wahl in Frankreich ist also auf eine Kombination politischer (Verweigerung einer Anpassung der Wahlkreiseinteilung) und geografischer Gründe (stärkere Repräsentation dünn besiedelter Gebiete durch Mindestrepräsentation) zurückzuführen.

\subsection{Norwegen}

Aus politischen und geografischen Gründen wird auch in Norwegen die Gleichheit bei der Wahl zum Storting, dem Parlament, verletzt. 150 der 169 Storting-Abgeordneten werden nach dem Verhältniswahlrecht in Wahlkreisen gewählt; 19 Ausgleichsmandate erhalten Parteien, die im Verhältnis zur Gesamtstimmenzahl zu wenig Sitze bekommen haben. Dabei wird in jedem der 19 Verwaltungsbezirke (Fylke) ein Ausgleichsmandat vergeben. ${ }^{28}$ Jedes Fylke bildet einen Mehrpersonenwahlkreis und entsendet seiner Größe entsprechend eine bestimmte Zahl von Abgeordneten. Unter „Größe“ wird bei der Wahlkreiseinteilung neben der Bevölkerungszahl die Fläche eines Fylke verstanden ${ }^{29}$, damit die dünn besiedelten, weit von der Hauptstadt entfernten Gebiete durch verhältnismäßig mehr Mandate stärker repräsentiert sind als die zentral gelegenen, dichter besiedelten Fylker im Süden und Osten. ${ }^{30}$ So

24 Vgl. ebenda. Zur Verletzung der Wahlrechtsgleichheit in Frankreich siehe auch Michel Balinski, Lois électorales. Le vote à la découpe, in: Commentaire, 28. Jg. (2005), H. 110, S. 439 ff.

25 Vgl. Frédéric Rouvillois, a.a.O. (Fn. 21), S. 82.

26 Vgl. Michel Balinski, Le suffrage n'est plus universel, a.a.O. (Fn. 23).

27 Vgl. Assemblée Nationale, Élections des députés, in: dies., Textes relatifs aux POUVOIRS PUBLICS, Constitution. - Lois organiques, Textes législatifs et réglementaires, 11. Auflage, o.O. 2007, S. V-1 ff., unter http://www.assemblee-nationale.fr/connaissance/pouvoirs-publics/pouvoirs_publics.pdf, abgerufen am 30. Mai 2007.

28 Eine ähnliche Regelung zur gerechten geografischen Mandatsverteilung hat Schweden. Jeder der 310 Wahlkreise entsendet unabhängig von der Wahlberechtigtenzahl einen Abgeordneten; daher sind in Wahlkreisen mit hoher Bevölkerungsdichte mehr Stimmen für einen Sitz nötig als in dünn besiedelten Gebieten; zur Herstellung der Proportionalität dienen 39 Ausgleichsmandate. Die dünn besiedelten Gebiete sind anders als in Norwegen damit nicht überrepräsentiert, aber doch mit mehr Gewicht vertreten, als es in Deutschland der Fall wäre. Vgl. Britta Glißsman, Zentralstaat versus Regionen, Frankfurt am Main 2004, S. 60.

29 „The number of members to be returned from each constituency depends on the population and area of the county. Each inhabitant counts one point, while each square kilometre counts 1.8 points." (http://odin.dep.no/krd/valg2005/english/016051-991372/dok-bn.html, abgerufen am 30. November 2005) Die Zahl der Parlamentssitze wird alle acht Jahre neu berechnet. Die heutige Regelung gilt auch für die nächste Stortingwahl 2009. Danach wird wieder eine Neujustierung der Wahlkreise erfolgen.

30 Auch Dänemark berücksichtigt bei der Verteilung der Mandate auf die einzelnen Mehrpersonenwahlkreise die Zahl der Einwohner und die geografische Größe eines Wahlkreises, zusätzlich aber 
entsendet das im Norden gelegene, dünn besiedelte, aber geografisch größte Fylke Finnmark fünf Abgeordnete, obwohl ihm aufgrund der geringen Bevölkerungszahl nur zwei Mandate zustünden. Das bevölkerungsreichste, aber flächenmäßig kleinste Fylke Oslo bestimmt hingegen nur 17 statt 22 Abgeordnete ${ }^{31}$; daher zählt die Stimme eines Wählers in Finnmark doppelt so viel wie in Oslo, nämlich knapp 15.000 gegenüber gut 30.000 Stimmen.

Gründe für die Überrepräsentation der dünn besiedelten Gebiete liegen auf der Hand: Drei Viertel der norwegischen Gesamtbevölkerung leben im Süden. Auch die größten städtischen Ballungszentren (Oslo, Bergen, Trondheim) und die modernen Industrie- und Dienstleistungsbetriebe liegen dort. Den Norden zeichnen vor allem extrem geringe Besiedlung und schwierige Lebensbedingungen aus. Die Stabilisierung seiner Besiedlung und die Wahrung möglichst gleicher Lebensverhältnisse im ganzen Land haben daher höchste Priorität. Die Wahlrechtsregelungen zur Überrepräsentation der entlegenen Fylker gehören zur „Distriktspolitik“: Sie sollen größeren Einfluss im Storting haben, um dem weiteren wirtschaftlichen und soziokulturellen Auseinanderdriften von Nord und Süd entgegenzuwirken. ${ }^{32}$ So wird die Praxis der Wahlkreiseinteilung auch entsprechend begründet: „We still think that the urban towns have stronger access to respond than the less populated and huge areas without the corresponding infrastructure." 33

\subsection{Wahlen zum Europäischen Parlament (EP)}

Ähnlich wie im Vereinigten Königreich führt die Verteilung der derzeit 785 Sitze im EP auf die 27 Mitgliedstaaten zu extrem unterschiedlichen Zählwerten der Stimmen, da die Mandate nicht proportional nach dem Anteil an der EU-Gesamtbevölkerung verteilt sind. Jeder Mitgliedstaat hat eine in Artikel 190 Abs. 2 EGV festgelegte Zahl von Sitzen mit einer Mindestrepräsentation von vier. Durch diese Kontingentierung sind die bevölkerungsreichen Mitgliedstaaten unterrepräsentiert, die mit geringer Bevölkerung stark überrepräsentiert. Deutschland als bevölkerungsreichstes Mitglied (18,18 Prozent der EU-Bevölkerung) hat 99 Mandate (12,61 Prozent der EP-Sitze), Malta als kleinster Mitgliedstaat (0,09 Prozent der Bevölkerung) fünf (0,63 Prozent der EP-Sitze), Luxemburg mit nur wenig mehr Einwohnern als Malta sechs. Auf einen deutschen Abgeordneten kommen demnach 828.000 Einwohner, auf seinen luxemburgischen Kollegen nur 79.000. Die Stimme eines luxemburgischen Wählers hat also mehr als zehnmal soviel Gewicht wie die eines Deutschen.

noch die Anzahl der Wähler bei der letzten Parlamentswahl. Vgl. $\$ 31$ Abs. 3 der dänischen Verfassung sowie $\$ 10$ Abs. 2 des dänischen Wahlgesetzes.

31 Finnmark hat auf $48.637 \mathrm{~km}^{2}$ nur 73.210 Einwohner, Oslo auf $454 \mathrm{~km}^{2} 521.886$ Einwohner (2004); vgl. Statistics Norways unter http://www.ssb.no/english/subjects/00/minifakta_en/ty/index.html, abgerufen am 30. November 2005. Die Berechnung, wie viele Mandate den Fylke ohne Gebietsfaktor zustehen würden, teilte Torodd Noreng vom Informationsdienst des Storting der Autorin per E-Mail mit.

32 Vgl. Auswärtiges Amt, Norwegen, Innenpolitik, unter http://www.auswaertiges-amt.de/www/de/ laenderinfos/laender/laender_ausgabe_html?type_id=10\&land_id=126, abgerufen am 10. November 2005.

33 So Torodd Noreng vom Informationsdienst des Storting in einer E-Mail an die Autorin. Vgl. auch Max-Planck-Institut für ausländisches öffentliches Recht und Völkerrecht, a.a.O. (Fn. 5), S. 661. 
Für diese Mandatskontingentierung sind mehrere Gründe zu nennen: Erstens ist auch den kleineren bevölkerungsschwachen Staaten eine Mindestrepräsentation zuzusichern, die ihren Parteienpluralismus widerspiegeln kann. Zweitens würde die Abgeordnetenzahl „alle vernünftigen Dimensionen" 34 sprengen und eine effektive Parlamentsarbeit behindern, würde den großen Ländern proportionale Repräsentation eingeräumt. Wandte man etwa das Verhältnis 80.000 Einwohner pro Sitz (Malta) auf Deutschland an, stünden ihm allein 1025 Mandate zu, Frankreich 776, dem Vereinigten Königreich 747. So sind die Mandatskontingente in einer EU mit 27 Mitgliedstaaten aus praktischen und politischen Überlegungen unvermeidbar. Allerdings wäre diese Verletzung der Wahlrechtsgleichheit in Anbetracht der Debatte um das Demokratiedefizit in der EU eher vertretbar, gäbe es ein einheitliches Wahlrecht zum EP.

\subsection{Bewertung}

Der Verstoß gegen den Gleichheitsgrundsatz bei der Wahlkreiseinteilung erfolgt aus politischen und räumlichen Gründen und in der Regel nicht willkürlich. Den dünn besiedelten ländlichen Gebieten wird eine größere Repräsentation eingeräumt, um ihren Einfluss im nationalen Parlament zu verstärken. ${ }^{35}$ Dies erscheint wegen der Dominanz der urbanen Ballungsgebiete sinnvoll: Metropolenferne Gebiete haben meist eine schlechtere Infrastruktur, werden bei politischen und ökonomischen Entscheidungen oft vernachlässigt und in Zeiten knapper Kassen mit ihren Forderungen und Problemen von den nationalen Parlamenten kaum gehört. Mit der stärkeren Repräsentation kann auch politisch ein Zeichen gesetzt und der Bevölkerung das Gefühl vermittelt werden, ernst genommen und durch stärkeren Einfluss im nationalen Parlament für die Probleme, die das Leben in strukturschwachen, dünn besiedelten Gebieten zwangsläufig mit sich bringt, entschädigt zu werden.

\section{Ein Modell für die Bundesrepublik Deutschland?}

Diskussionsbedürftig ist, ob eine Wahlkreiseinteilung, die bestimmten Bevölkerungsgruppen größere Repräsentation im Parlament gibt, auch in Deutschland Anklang finden kann. Hier soll das norwegische System der Wahlkreiseinteilung als Modell dienen, da die Überrepräsentation der dünn besiedelten Gebiete und die damit verbundene Verletzung der Wahlrechtsgleichheit erstens verständlich ist und zweitens die Überrepräsentierung nicht willkürlich, sondern nach einer festgelegten Formel erfolgt. Es könnte vor allem dort Vorbild für Deutschland sein, wo wie in Norwegen die Bevölkerung und Landesentwicklung nicht gleichmäßig verteilt sind.

Dies soll nachfolgend anhand eines konkreten Beispiels - der Überrepräsentation dünn besiedelter Gebiete in Berlin-Brandenburg - diskutiert werden. Ausgewählt wurde dieses Beispiel, weil Brandenburg einerseits flächenmäßig das größte ostdeutsche Bundesland ist, andererseits aber mit die geringste (und schrumpfende) Bevölkerung aufweist. Interessant ist der Fall insbesondere, weil bei der geplanten Fusion Ende der 1990er Jahre Brandenburg

34 Dieter Nohlen, a.a.O. (Fn. 4), S. 398.

35 Vgl. ebenda, S. 87 f. 
auf das stark besiedelte Berlin getroffen wäre. Bevölkerung und Landesentwicklung wären nicht gleichmäßig verteilt gewesen. Die Fusion scheiterte auch deshalb am negativen Votum der Bürger Brandenburgs, die eine Dominanz Berlins befürchteten. ${ }^{36}$ Hätte das Scheitern durch eine „norwegische“ Wahlkreiseinteilung verhindert werden können?

\subsection{Demografischer Wandel und das Problem dünn besiedelter Flächen}

In mehreren Bundesländern sind Bevölkerung und Landesentwicklung nicht gleichmäßig verteilt, beispielsweise in Niedersachsen oder Bayern. Besonders aber in den neuen Bundesländern ist dies zu beobachten. Der Bevölkerungsrückgang, speziell in den dünn besiedelten Flächen, verschärft die bestehenden Ungleichheiten. Mecklenburg-Vorpommern, Brandenburg, Sachsen-Anhalt und Thüringen sind bundesweit die Länder mit der geringsten Bevölkerungsdichte; in Regionen wie den Landkreisen Prignitz und Uckermark (Brandenburg) oder der Altmark (Sachsen-Anhalt) werden für das Jahr 2015 Bevölkerungsdichten zwischen 40 und 45 Einwohner je Quadratkilometer erwartet. „Dieser Trend der Schrumpfung ist in vielen Regionen angesichts der demographischen Fakten und der begrenzten politischen Handlungsspielräume in absehbarer Zeit nicht mehr umzukehren. “37

Der tiefgreifende demografische Wandel betrifft alle Lebensbereiche und hat Auswirkungen auf Wirtschaft, Politik und Gesellschaft. So sind die sozialen Sicherungssysteme und die soziale Infrastruktur wie die Bereitstellung von Kinderbetreuung, Beratungsdiensten und Pflegeheimen schon jetzt vor neue Herausforderungen gestellt. Zudem haben die wenig besiedelten Räume meist mit ökonomischen Problemen zu kämpfen: Arbeitsplatzabbau, Abwanderung junger Menschen in ökonomisch aussichtsreichere Gebiete usw. Meist ziehen „die regelmäßig bevölkerungsreicheren, starken, meist boomenden Landesteile die letzten Ressourcen aus den zudem in der Regel dünn(er) besiedelten Gebieten“38.

\subsection{Die Besonderheit Brandenburgs}

Besonders das größte ostdeutsche Flächenland hat mit den Folgen des demografischen Wandels zu kämpfen. Mit circa 2,6 Millionen Einwohnern auf knapp 30.000 Quadratkilometern ist es dünn besiedelt und vorwiegend agrarisch geprägt. An der Peripherie finden sich bevölkerungsarme und strukturschwache Regionen wie die Prignitz oder Uckermark. Im Zentrum liegt inselförmig der Stadtstaat Berlin. Im „Speckgürtel“ rund um Berlin leben inzwischen 39 Prozent der Brandenburger. ${ }^{39}$ Diese Stadtrandzone mit den Städten Potsdam, Ludwigsfelde, Teltow und Wildau ist das einzige Gebiet mit kontinuierlichem Bevöl-

36 Vgl. zum Volksentscheid auch Otmar Jung, Die Volksabstimmung über die Länderfusion BerlinBrandenburg: Was hat sich bewährt - wer ist gescheitert?, in: ZParl, 28. Jg. (1997), H. 1, S. 13 ff.

37 Vgl. Martina Kocks, Anpassungsstrategien für ländliche/periphere Regionen mit starkem Bevölkerungsrückgang in den neuen Ländern, in: Werkstatt Praxis, Nr. 38/2005, S. 4.

38 Erich Röper, Länderneugliederung löst kein Problem strukturschwacher Räume. Eine Replik auf den Beitrag von Christian Stolorz in Heft 2/1997 der ZParl, in: ZParl, 28. Jg. (1997), H. 4, S. 722 ff., S. 723.

$39 \mathrm{Vgl}$. Andrea Beyerlein, Brandenburger zieht es in Berliner Umland, in: Berliner Zeitung vom 1. Dezember 2005, unter https://www.berlinonline.de/berliner-zeitung/brandenburg/505173.html, abgerufen am 19. Dezember 2005. 
kerungswachstum seit $1995^{40}$; auch die Geburtenraten sind hier deutlich höher als im übrigen Land. ${ }^{41}$ Die meisten Regionen Brandenburgs und vor allem die zentrenfernen Gebiete haben mit starker Abwanderung nach Westdeutschland und sehr geringem Geburtenniveau zu kämpfen. ${ }^{42}$ Das wirtschaftliche Gefälle des Speckgürtels um Berlin zu den Randgebieten ist noch stärker als das Bevölkerungsgefälle, und die Bevölkerungsentwicklung wird die Disparitäten weiter verstärken. Die Folgen für öffentliche Infrastruktur und wirtschaftliche Entwicklung sind sichtbar: Wohnungen stehen leer, Schulen und Kindertagesstätten müssen geschlossen werden, Personennahverkehr und Kulturangebote geraten unter Druck, Arbeitsplätze werden abgebaut. ${ }^{43}$ Die Menschen in den Gebieten mit deutlichen Peripherisierungs-, Schrumpfungs- und Alterungsprozessen „haben das Gefühl, in Vergessenheit zu geraten, keine politische Aufmerksamkeit zu erfahren: abgeschrieben zu sein “44.

\subsection{Länderneugliederung als Lösung?}

Eine Fusion von Berlin und Brandenburg wird seit der deutschen Wiedervereinigung regelmäßig diskutiert und könnte eine Verbesserung der Situation der dünn besiedelten Gebiete mit sich bringen. Bei dem 1996 gescheiterten Versuch wäre unter anderem die Herstellung gleichwertiger Lebensbedingungen ein Staatsziel des neuen Bundeslandes geworden. ${ }^{45} \mathrm{Zu}-$ dem erhoffte man sich durch die Fusion mehr Wettbewerbsfähigkeit der Region, neue Wachstumsimpulse und Arbeitsplätze sowie durch den Abbau von Doppelverwaltung die Einsparung von Kosten. ${ }^{46}$ Das Vermeiden des teuren Nebeneinanders hätte Ressourcen frei gesetzt, die für die Stärkung der regionalen Wirtschaft und die Verbesserung der Infrastruktur zur Verfügung gestanden hätten.

Die Mehrheit der Bürger Berlins (53,6 Prozent) befürwortete in der Volksabstimmung am 5. Mai 1996 die Bildung des gemeinsamen Bundeslands. Dies scheiterte jedoch am negativen Votum (62,7 Prozent) der Brandenburger Bevölkerung. Eine bedeutende Rolle spielte dabei die Furcht vor einer Dominanz der zentrenfernen Entwicklungsräume durch Berlin und der damit verbundene Verlust der Eigenständigkeit Brandenburgs. Auch Berlins katastrophale

40 Bis 2030 wird das Berliner Umland um weitere 54.000 Einwohner wachsen, die Randgebiete werden 224.000 Einwohner verlieren. Vgl. Landesregierung Brandenburg Staatskanzlei, Demografischer Wandel in Brandenburg. Erneuerung aus eigener Kraft, 2. Bericht der Landesregierung zum demografischen Wandel, Potsdam 2005, S. 4 ff., unter http://www.brandenburg.de/cms/media.php/1168/db_end.pdf, abgerufen am 28. November 2005.

41 Fast die Hälfte der gut 18.000 Geburten entfiel 2004 auf die Stadtrandzone von Berlin; vgl. Andrea Beyerlein, a.a.O. (Fn. 39).

42 Vgl. Christiane Buchner / Jochen Franzke, Das Land Brandenburg. Kleine politische Landeskunde, Potsdam 2002.

43 Vgl. Matthias Platzeck, Bevölkerungsrückgang erfordert kreative Lösungen, in: Allgemeine Zeitung Mainz vom 25. Oktober 2005, unter: www.brandenburg.de/sixcms/detail.php?id=108041 \&_siteid=15, abgerufen am 28. November 2005.

44 Stephan Beetz, Ländliche Politik im demografischen Wandel, in: APuZ, B $21 / 22$ (2006), S. 25 ff.

45 Neben der Betonung dieses Staatsziels in der Präambel enthält Kapitel V des Neugliederungsstaatsvertrags eine Reihe von Vereinbarungen zur Landesentwicklung und Schaffung gleichwertiger Lebensverhältnisse. Parallel dazu vereinbarten Berlin und Brandenburg einen Landesplanungsvertrag. Er hat auch über die Ablehnung des Neugliederungsvertrags hinaus Bestand.

46 Vgl. Christiane Buchner / Jochen Franzke, a.a.O. (Fn. 42), S. 38; Herbert Schwenk, Ein Dualismus mit langer Tradition, in: Berlinische Monatsschrift, 10. Jg. (2001), H. 7, S. 89 ff., S. 91. 
Haushaltslage war für viele ein Grund, die „Länderehe“ abzulehnen. Nach dem Scheitern des Neugliederungsprojekts wurden neue Absichtserklärungen für einen zweiten Versuch abgegeben. Die Regierungschefs Manfred Stolpe und Eberhard Diepgen vereinbarten, 2006 eine neue Volksabstimmung abzuhalten. ${ }^{47}$ Den Termin strich die 2004 ins Amt gekommene Brandenburger Regierung aus Sorge vor einem erneuten Scheitern jedoch aus dem Koalitionsvertrag. Der Zusammenschluss Berlins und Brandenburgs rückt in weite Ferne. ${ }^{48}$

\subsection{Erfolg durch Wahlkreiseinteilung?}

Die Gründe für die Ablehnung der Fusion durch die Brandenburger Bevölkerung sind nachvollziehbar; die Unterschiede der wenig besiedelten zentrenfernen Entwicklungsräume Brandenburgs und der Metropole Berlin liegen auf der Hand und damit auch die Gefahr, dass das Flächenland vom Stadtstaat dominiert wird. Ebenso offensichtlich ist aber auch eine Reihe von Vorteilen einer Länderfusion. Vor diesem Hintergrund sollte über die Möglichkeit nachgedacht werden, in einem Bundesland Berlin-Brandenburg die Wahlkreise zugunsten der Entwicklungsräume einzuteilen. Norwegen könnte als Modell dienen. Auch dort gibt es den Gegensatz dünn besiedelter, agrarisch geprägter Räume zu wirtschaftlich starken, dicht besiedelten Metropolenregionen. Die Wahrung möglichst gleicher Lebensverhältnisse ist ein Ziel der norwegischen Raumpolitik; die stärkere Repräsentation der Entwicklungsräume bei den Wahlen zählt hierzu. Dafür würden die Wahlkreise in dünn besiedelten Gebieten deutlich weniger Wahlberechtigte umfassen als in stärker besiedelten Landesteilen, das heißt für die deutsche Praxis, dass in begründeten Fällen von der Toleranzgrenze von 25 Prozent abgewichen werden könnte. Eine weitere Möglichkeit wäre die Erweiterung des Parlaments unter Beibehaltung der Toleranzgrenze um einige Mandate, bei deren Besetzung die Bevölkerungsdichte der einzelnen Wahlkreise berücksichtigt würde. ${ }^{49}$

Viel spräche dafür, ein solches Modell für ein mögliches Land Berlin-Brandenburg zu erproben. Erich Röper unterstreicht zur „Länderehe“, dass die „Entwicklung strukturschwacher Gebiete [...] nur möglich [ist], wenn die politischen Entscheidungen über die nötigen Maßnahmen dort fallen "50. So könnte eine stärkere Repräsentation der dünn besiedelten Gebiete Brandenburgs durch eine Wahlkreiseinteilung entgegen der Wahlrechtsgleichheit das weitere wirtschaftliche und soziokulturelle Auseinanderdriften von Speckgürtel und Entwicklungsräumen verhindern. Bevölkerung und Abgeordnete könnten besser auf ihre Probleme aufmerksam machen und hätten eher das Gefühl, trotz wirtschaftlicher Schwäche und Entfernung zum (wirtschaftlichen und politischen Entscheidungs-) Zentrum Einfluss ausüben zu können. Bei richtiger Information und Kommunikation dürften

47 Vgl. Herbert Schwenk, a.a.O. (Fn. 46), S. 99.

48 Die Koalitionsvereinbarung enthält statt des noch vor der Wahl vorgesehenen Fusionstermins nur das „Bekenntnis zum Zusammenschluss“; vgl. Martin Peter, Mehrheit der Bürger für Fusion, in: Das Parlament, Nr. 52-53/2004, unter http://www.das-parlament.de/2004/52-53/Inland/005. html, abgerufen am 28. November 2005.

49 Da die Einführung eines solchen Modells weit reichende rechtliche und mathematische Implikationen mit sich brächte, kann es hier nicht um eine Konkretisierung gehen. Das Beispiel soll aber zeigen, dass eine stärkere Berücksichtigung der Bevölkerung in dünn besiedelten Gebieten ohne einen Systemwechsel im deutschen Wahlsystem durchaus möglich wäre.

50 Erich Röper, Länderneugliederung löst kein Problem strukturschwacher Probleme, a.a.O. (Fn. 38), S. 723. 
die Bürger von dem Modell zu überzeugen sein. So könnte sich die Ablehnung der Fusion aus Angst vor Berlins Dominanz in Zustimmung wenden, wüssten sie, dass die dünn besiedelten Regionen in einem neuen Bundesland nicht „untergehen“.

Allerdings ist fraglich, ob die Bewohner der dicht besiedelten Gebiete die stärkere Repräsentation der strukturschwachen Ränder akzeptieren und auf einen Teil ihrer Souveränität zugunsten der Mitbürger dort verzichten würden. Auch ist unklar, ob die politischen Eliten das „,norwegische Modell“ der Wahlkreiseinteilung nach den Ergebnissen der letzten Landtagswahlen in Sachsen und Brandenburg mit deutlichen Stimmengewinnen für NPD und DVU vor allem in strukturschwachen Gebieten unterstützen. Dennoch sollte die stärkere Repräsentation der Randgebiete Brandenburgs von Politik und Bevölkerung diskutiert werden. Vielleicht könnte dies dem Projekt des Zusammenschlusses der zwei Bundesländer neuen Schwung geben.

\section{Gewichtetes Wahlrecht zum Ausgleich von Entwicklungsdefiziten}

Eine Verletzung des Grundsatzes der Wahlrechtsgleichheit kann in bestimmten Fällen akzeptiert werden. Bei der Wahlkreiseinteilung gibt es ,politische Argumente, die als rechtens anerkannt werden und die es folglich gestatten, vom Gleichheitsgrundsatz abzuweichen " 51 . Im Vereinigten Königreich wurden auf diese Weise Randgebiete an England gebunden, und ihre überproportionale Repräsentation galt als Ersatz für Regionalparlamente. In Spanien erhalten kleine Wahlkreise durch die Mindestrepräsentation von drei Mandaten mehr Gewicht; ähnlich sieht es in Frankreich aus, wo jedem Département eine Mindestrepräsentation von zwei Mandaten gewährt wird. In Norwegen werden die dünn besiedelten, strukturschwachen Gebiete stärker im Parlament vertreten, indem bei der Verteilung der Mandate auf die 19 Mehrpersonenwahlkreise neben der Bevölkerungszahl ihre geografische Lage und flächenmäßige Größe berücksichtigt werden. Schließlich sind bei den Wahlen zum Europäischen Parlament die kleineren Mitgliedstaaten durch die Mandatskontingentierung überrepräsentiert; die Garantie eines Grundmandats von vier Sitzen soll nicht nur den Parteienpluralismus in diesen Ländern widerspiegeln, sondern ihnen gegenüber den großen Mitgliedstaaten die Chance auf Berücksichtigung ihrer Interessen geben.

In Deutschland wären solche Verletzungen der Wahlrechtsgleichheit rechtlich noch nicht möglich, obwohl eine die dünn besiedelten Gebiete stärker repräsentierende Wahlkreiseinteilung in Bundesländern sinnvoll wäre, wo Landesentwicklung und Bevölkerung nicht gleichmäßig verteilt sind. Die gescheiterte Fusion Berlin und Brandenburgs zeigt, dass die Bürger ein Gespür für solche Unterschiede und die Dominanz boomender Metropolenregionen über bevölkerungsarme, strukturschwache Gebiete haben. Eine Wahlkreiseinteilung nach „norwegischem Modell“ könnte ihre Probleme nicht lösen, wäre aber ein erster Schritt zu möglichst gleichen Lebensverhältnissen in einem Bundesland. Die Diskussion einer solchen Wahlkreiseinteilung ist daher wünschenswert.

51 Dieter Nohlen, a.a.O. (Fn. 4), S. 87. Der Gedanke findet sich auch im Bundesverfassungsgerichtsurteil vom 11. November 1999 zum Länderfinanzausgleich, indem der Einwohnerwert der dünn besiedelten Gebiete ähnlich den Stadtstaaten „veredelt“ werden könne; vgl. BVerfGE 101, S. 158 ff.; dazu Helmut Seitz, Der Einfluss der Bevölkerungsdichte auf die Kosten der öffentlichen Leistungserstellung, Gutachten im Auftrag der Finanzministerien der Länder Mecklenburg-Vorpommern und Brandenburg, Frankfurt an der Oder 2000. 